\title{
The haemodynamic and ventilatory responses with I-gel, laryngeal mask airway and tracheal intubation during laparoscopic cholecystectomy
}

Maharjan SK

Associate Professor, Department of Anaesthesiology and Intensive Care, Kathmandu Medical College Teaching Hospital,

Kathmandu, Nepal

\section{Abstract}

Background: Despite use of adequate medications and techniques, tracheal intubation induces haemodynamic stress response, which can be minimized by using supraglottic airway devices instead of tracheal tube in elective surgical cases with adequate oxygenation and ventilation.

Objectives: To compare haemodynamic variables and ventilation parameters of I-gel and laryngeal mask airway with tracheal intubation during laparoscopic surgery.

Methods: This is a prospective randomized comparative study among 90 cases of American Society of Anesthesiologists physical status class I and II, undergoing laparoscopic cholecystectomy, who were equally divided into three groups of 30 patients each: I-gel group, Laryngeal mask airway group and Tracheal tube group. Randomization was done with pick up of cards from sealed envelope.

Basal readings of heart rate, systolic, diastolic and mean arterial pressure were recorded and these parameters were measured again before airway device placement, one, three and five minutes after airway manipulation, during carboperitoneum creation and before and after extubation. Oxygen saturation, end tidal $\mathrm{CO}_{2}$, airway pressure and inhaled and exhaled tidal volume and minute volume were monitored before, during and after carboperitoneum creation. Leak volume was calculated by deducing exhaled tidal volume from inhaled tidal volume. Statistical analysis (Analysis of variance test) was done to see the differences among the groups.

Results: Haemodynamic perturbations were maximum with tracheal intubation and moderate with laryngeal mask airway while stable haemodynamics was observed with I-gel. Intra and inter-group comparison revealed significant differences after use of airway devices and after removal as well.

Regarding ventilatory response, oxygenation and ventilation was well maintained with maximum airway pressure of mean \pm SD: $20.11 \pm 3.46,20.24 \pm 4.42,19.05 \pm 4.82 \mathrm{cmH}_{2} \mathrm{O}$ during carboperitoneum creation in I-gel, laryngeal mask airway and tracheal tube group respectively and oxygen saturation of 98 to $100 \%$ and end tidal $\mathrm{CO}_{2}$ level of $31-35 \mathrm{mmHg}$. In all groups, minute volume was well maintained and leak volume of $18.88 \pm 12.40,17.13 \pm 13.32$ and $20.89 \pm 12.20 \mathrm{ml}$ were recorded in I-gel, laryngeal mask airway and tracheal tube group respectively during carboperitoneum creation. There was no statistically significant difference among the groups at any time regarding the monitored parameters.

Conclusion: Among the three airway management devices used during general anaesthesia with positive pressure ventilation, l-gel produced least haemodynamic stress response, both supraglottic devices can be used with proper size and placement with acceptable haemodynamics and ventilation during laparoscopic surgery.

Key words: Haemodynamic changes, I-gel, laparoscopy, laryngeal mask airway, positive pressure ventilation, tracheal intubation.

\section{INTRODUCTION}

$\Delta$ dministration and maintenance of general anaesthesia necessitates tracheal intubation in most

Address for correspondence

Dr. Shyam Krishna Maharjan

Associate Professor, Department of Anaesthesiology and Intensive Care

Kathmandu Medical College Teaching Hospital, Kathmandu

E-mail: shyammaharjan2@hotmail.com of the cases but the procedure is not without adverse effects. Among them, haemodynamic perturbations are of major concern to us during intra operative period. To minimize these effects, supraglottic airway devices like laryngeal mask airway (LMA) or I-gel can be used instead of tracheal intubation in selected elective cases.

There are several well established advantages of supraglottic airway devices compared to a tracheal 
The haemodynamic and ventilatory responses with I-gel, laryngeal mask airway and tracheal intubation during ....

tube like less haemodynamic stress response, lower incidence of sore throat and increased case turnover'. Laryngeal mask airways are being used for that purpose since 1990's and recently l-gel, a new supraglottic airway device is added to that list. Different reports state that classic LMA or proseal LMA and I-gel can be used for management of airway during anaesthesia with positive pressure ventilation ${ }^{2-4}$.

Despite use of strong opioids, adequate anaesthetic depth and application of other stress reducing techniques and agents, there is release of catecholamines and cortisol which causes increase in heart rate and pressor response after pharyngeal and laryngotracheal manipulation, usually more after laryngoscopy and tracheal intubation ${ }^{2,5,6}$.

These responses may be of less importance in American Society of Anesthesiologists (ASA) physical status-I patients but may be life threatening to patients with hypertension, diabetes mellitus, ischemic heart disease and cerebrovascular diseases. With the use of LMA or l-gel, these effects can be decreased during general anaesthesia with mechanical ventilation with adequate oxygenation and ventilation ${ }^{7-9}$.

Different studies have shown that I-gel and classic or proseal LMA can be used safely during positive pressure ventilation with stable haemodynamics and normal oxygenation and ventilation ${ }^{8,10,11}$. Most of the studies have been done in non-obese patients but there is one report of safe use of I gel or LMA in moderate obesity as well ${ }^{12}$. There are reports of adequate seal during moderate airway pressure by these supraglottic devices and gastric distension similar to tracheal intubation ${ }^{13,14}$.

This study was designed to assess the haemodynamic stress response and ventilation parameters with the use of tracheal tube, classic LMA and I-gel during laparoscopic surgery where carboperitoneum is created with increase in intraabdominal pressure.

\section{METHODS}

This is a randomized, prospective interventional study in patients attending for laparoscopic cholecystectomy in Kathmandu Medical College Teaching Hospital from March 2012 to May 2012. After approval from the institute's ethical committee, 90 patients (both male and females) of ASA physical status I and II, of age 20-65 years undergoing laparoscopic cholecystectomy under general anaesthesia with positive pressure ventilation were taken for study.
Sample size calculation was done with previous study results of means and standard deviation and considering a reduction of 15.0 relative to control value in mean heart rate during the use of airway device. The required sample size came out to be 30 patients per group keeping power at $80 \%$ and alpha level of 0.056 . They were equally divided into three groups, I-gel, LMA and Tracheal tube group of 30 each by randomly selected envelops.

Emergency cases, hypertensive cases and potential difficult airway cases were excluded from the study. Patients with body mass index more than $30 \mathrm{~kg} / \mathrm{m}^{2}$, hiatus hernia and gastro-oesophageal reflux disease were also excluded.

After careful evaluation and airway assessment, patients were premedicated with Diazepam, Ranitidine and Metoclopromide the evening before and in the morning of the day of surgery. Consent and fasting status were confirmed and an intravenous line was opened, routine monitors applied and baseline heart rate, arterial pressure and oxygen saturation recorded. Anaesthesia was induced with Propofol $2 \mathrm{mg}$ per kg, Fentanyl 2 microgram per kg and airway placement was facilitated by Vecuronium $0.1 \mathrm{mg}$ per $\mathrm{kg}$.

Decision of airway placement was done by sealed envelope method randomly and airway placement was done by experienced anaesthesiologist. Size of airway devices used were: tracheal tube $7.0 \mathrm{~mm}$ internal diameter for females and $8.0 \mathrm{~mm}$ internal diameter for males, classic LMA: size 4.0 for females and size 5.0 for males and I gel: size 4.0 for both males and females, as recommended by the manufacturer. Difficult intubation cases and more than one attempt of airway manipulation were also excluded from the study. Anaesthesia was maintained with $1-2 \%$ Isoflurane, $50 \%$ oxygen in air and positive pressure ventilation.

Surgical incision was requested after five minutes of induction and airway manipulation to avoid likely stimuli. Serial heart rate, systolic, diastolic and mean arterial pressure, end tidal carbon dioxide tension and oxygen saturation were recorded after induction, one, three and five minutes after airway device placement, during carboperitoneum period and before and one minute after removal of the airway device.

All patients were ventilated with $8-10 \mathrm{ml}$ per $\mathrm{kg}$ of tidal volume to maintain end tidal $\mathrm{CO}_{2}$ in the range of 30-40 $\mathrm{mmHg}$. During carboperitoneum, the intra-abdominal 
pressure was maintained between 12-14 $\mathrm{mmHg}$. Set or inhaled tidal volume, exhaled tidal volume and minute volume was recorded before during and after carboperitoneum and leak volume was calculated by deducting exhaled volume from inhaled tidal volume.

After completion of surgery, neuromuscular blockade was reversed with recommended dose of Neostigmine and Glycopyrrolate and after adequate respiratory efforts, airway devices were removed and when patients were fully awake and responding to verbal commands, they were transferred to postoperative ward for further management.

Statistical analysis was done with Statistical Package for Social Sciences (SPSS) for windows version 17. Significance test was done with one way Analysis of Variance (ANOVA) test. Results were displayed as mean \pm standard deviation and $p$ value less than 0.05 was taken as significant.

\section{RESULTS}

Patient characteristics regarding age, gender, weight and height were similar in the three groups. There was no significant difference among the groups regarding duration of anaesthesia and surgery as well (table 1).

\section{The Haemodynamic Response}

There was increase in mean heart rate after use of airway device in all three groups but maximum with tracheal tube and least with I-gel. While basal heart rate and heart rate prior to airway placement were comparable among all three groups, there was significant difference among the groups after one minute of use of airway device and after removal of the device ( $p$ value $<0.001$ and 0.021 respectively). Heart rate increment was also there after three and five minutes of airway manipulation but the difference among three groups was statistically not significant.

Systolic blood pressure (SBP) increment was also maximum with tracheal intubation, moderate with LMA and least with I-gel. Here again starting with similar baseline and prior to placement readings, there was highly significant difference in SBP among the groups after one minute of airway placement and one minute after removal as well ( $p=0.002$ and $<0.001$ respectively). Comparisons of systolic blood pressure in other time intervals in three groups were statistically not significant.
Regarding diastolic blood pressure, there was significant difference in one minute after insertion and after removal of airway device ( $p=0.019$ and 0.005$)$ but no significant difference detected in other time intervals studied. Though there was clinical increment in mean arterial pressure as well after manipulation of the airways, statistically, there was no significant difference observed (table 2).

When intra group comparison was made in heart rate, systolic blood pressure, there was increase in heart rate and systolic blood pressure in LMA and tracheal group with significant difference after intubation and extubation. But with I gel group of patients, the haemodynamics were stable and no significant increase was noted during the procedures (Table 2 ).

\section{The Ventilatory Response}

During the study period, airway pressure was well maintained during carboperitoneum with LMA and l-gel as well. The mean and standard deviation of maximum airway pressures recorded during carboperitoneum were $20.11 \pm 3.46,20.24 \pm 4.42$ and $19.05 \pm 4.82 \mathrm{cmH}_{2} \mathrm{O}$ for I-gel, LMA and tracheal tube respectively, which were similar ( $p=0.888)$. Oxygenation and ventilation was well maintained with all three airway devices. Oxygen saturation was maintained between 97 to 100 per cent and end tidal $\mathrm{CO}_{2}$ was maintained between 31 to 37 $\mathrm{mmHg}$ during the intraoperative period (table 4).

Ventilation during carboperitoneum was well maintained in all three groups with no audible leak during the maximum pressure attained and leak volume of 10 to $30 \mathrm{ml}$ with insignificant difference statistically ( $p=0.718$ ). Oxygen saturation during carboperitoneum period was maintained within 98 to $100 \%$ and end tidal $\mathrm{CO}_{2}$ of $31-35 \mathrm{mmHg}$ and there was no significant differences seen in mean exhaled tidal volume before, during and after carboperitoneum ( $p=0.482,0.387$ and 0.605 respectively) (table 3,4 ).

Regarding the use of supraglottic airway devices, I-gel insertion was 100 per cent successful, though there was some manipulation needed in six cases and during LMA use, failure was there in four cases and manipulation needed in ten cases. All tracheal tube intubation were in single attempt and no difficulty encountered during the study period. 
The haemodynamic and ventilatory responses with I-gel, laryngeal mask airway and tracheal intubation during ....

Table 1: Demographic characteristics, ASA physical status, airway status and duration of anaesthesia and surgery (mean \pm standard deviation) of the patients in three different groups.

\begin{tabular}{lcccc}
\hline Variables & I-gel group & LMA group & Tracheal tube group & p value \\
\hline Age (years) & $45.48 \pm 15.80$ & $45.44 \pm 15.11$ & $42.97 \pm 14.20$ & 0.749 \\
\hline Sex, Female:Male & $22: 8$ & $18: 12$ & $20: 10$ & 0.941 \\
\hline $\begin{array}{l}\text { Weight (kg) } \\
\text { Height (feet) }\end{array}$ & $57.16 \pm 8.80$ & $57.81 \pm 11.11$ & $58.67 \pm 10.50$ & 0.909 \\
\hline $\begin{array}{l}\text { Body Mass Index (kg/ } \\
\mathrm{m}^{2} \text { ) }\end{array}$ & $5.08 \pm 0.49$ & $4.90 \pm 0.48$ & $4.97 \pm 0.44$ & 0.382 \\
\hline $\begin{array}{l}\text { ASA status, I:II } \\
\text { Mallampati grade, I:II }\end{array}$ & 24.6 & 26.8 & 26.4 & 0.336 \\
\hline $\begin{array}{l}\text { Duration of } \\
\text { anaesthesia (minutes) }\end{array}$ & $23: 7$ & $20: 10$ & $24: 6$ & 0.165 \\
\hline $\begin{array}{l}\text { Duration of surgery } \\
\text { (minutes) }\end{array}$ & $50.17 \pm 15.64$ & $17: 13$ & $21: 9$ & 0.684 \\
\hline
\end{tabular}

$\mathrm{p}$ value calculated by ANOVA test.

Table 2: Haemodynamics (heart rate, systolic, diastolic and mean arterial pressure) observed during study period in three different groups.

\begin{tabular}{|c|c|c|c|c|c|c|c|c|}
\hline $\begin{array}{l}\text { Variables } \\
\text { (mean } \pm S D)\end{array}$ & Basal & $\begin{array}{c}\text { Before airway } \\
\text { placement }\end{array}$ & $\begin{array}{l}\text { After one } \\
\text { minute }\end{array}$ & $\begin{array}{l}\text { After three } \\
\text { minutes }\end{array}$ & $\begin{array}{l}\text { After five } \\
\text { minutes }\end{array}$ & $\begin{array}{c}\text { During } \\
\text { carboperitoneum }\end{array}$ & $\begin{array}{c}\text { Before } \\
\text { extubation }\end{array}$ & $\begin{array}{c}\text { After } \\
\text { extubation }\end{array}$ \\
\hline HR $^{*} 1$. & $81.58 \pm 14.22$ & $80.77 \pm 15.75$ & $79.23 \pm 13.31$ & $87.15 \pm 16.0$ & $85.03 \pm 15.38$ & $100.1 \pm 14.21$ & $86.77 \pm 17.27$ & $88.16 \pm 14.05$ \\
\hline 2. & $79.22 \pm 12.22$ & $85.96 \pm 17.11$ & $86.22 \pm 16.11$ & $86.81 \pm 16.5$ & $86.63 \pm 19.49$ & $98.00 \pm 12.90$ & $89.35 \pm 16.61$ & $95.79 \pm 17.17$ \\
\hline 3. & $79.03 \pm 14.49$ & $85.55 \pm 15.66$ & $98.45 \pm 13.57$ & $88.97 \pm 13.5$ & $86.03 \pm 13.42$ & $99.11 \pm 13.11$ & $91.61 \pm 17.23$ & $98.03 \pm 16.8$ \\
\hline$p$ value & 0.271 & 0.382 & 0.000 & 0.326 & 0.147 & 0.651 & 0.539 & 0.021 \\
\hline $\mathrm{SBP}^{+} 1$ & $120.8 \pm 16.26$ & $121.46 \pm 23.7$ & $112.03 \pm 22.7$ & $116.14 \pm 21.6$ & $130.0 \pm 12.1$ & $135.0 \pm 22.1$ & $134.2 \pm 23.1$ & $124.3 \pm 23.77$ \\
\hline 2. & $122.03 \pm 16.0$ & $127.78 \pm 24.9$ & $120.8 \pm 21.7$ & $116.2 \pm 20.58$ & $129.8 \pm 13.0$ & $131.8 \pm 23.0$ & $135.5 \pm 20.8$ & $137.7 \pm 20.0$ \\
\hline 3. & $117.7 \pm 17.34$ & $127.96 \pm 16.8$ & $132.5 \pm 26.03$ & $114.67 \pm 13.0$ & $132.9 \pm 17.1$ & $133.2 \pm 21.5$ & $134.6 \pm 16.3$ & $146.51 \pm 20.7$ \\
\hline$p$ value & 0.233 & 0.278 & 0.002 & 0.878 & 0.961 & 0.971 & 0.811 & 0.000 \\
\hline $\mathrm{DBP}^{\ddagger} 1$. & $82.67 \pm 14.31$ & $81.88 \pm 15.4$ & $77.14 \pm 10.05$ & $81.37 \pm 13.9$ & $92.7 \pm 15.9$ & $93.2 \pm 14.4$ & $85.2 \pm 14.31$ & $86.64 \pm 17.37$ \\
\hline 2. & $81.42 \pm 10.13$ & $84.03 \pm 13.32$ & $78.75 \pm 21.98$ & $81.70 \pm 17.7$ & $91.38 \pm 18.3$ & $89.03 \pm 14.6$ & $87.0 \pm 10.13$ & $91.60 \pm 13.89$ \\
\hline 3. & $82.78 \pm 13.8$ & $81.87 \pm 15.2$ & $88.71 \pm 19,2$ & $87.5 \pm 17.5$ & $89.7 \pm 13.5$ & $87.5 \pm 15.3$ & $88.2 \pm 13.8$ & $98.2 \pm 16.8$ \\
\hline$p$ value & 0.936 & 0.718 & 0.019 & 0.209 & 0.764 & 0.318 & 0.998 & 0.005 \\
\hline $\operatorname{MAP}^{\S} 1$. & $100.6 \pm 19.01$ & $93.40 \pm 23.8$ & $90.85 \pm 33.14$ & $102.62 \pm 23.9$ & $112.0 \pm 17.3$ & $115.8 \pm 18.7$ & $103.7 \pm 14.0$ & $105.60 \pm 28.1$ \\
\hline 2. & $100.28 \pm 16.5$ & $96.03 \pm 17.13$ & $96.53 \pm 18.97$ & $100.16 \pm 15.8$ & $107.8 \pm 16.2$ & $108.3 \pm 19.3$ & $111.0 \pm 14.6$ & $111.28 \pm 16.4$ \\
\hline 3. & $101.3 \pm 19.7$ & $100 . .9 \pm 17.3$ & $98.70 \pm 27.4$ & $105.0 \pm 14.2$ & $101.2 \pm 19.9$ & $107.1 \pm 22.9$ & $115.1 \pm 22.3$ & $117.15 \pm 21.0$ \\
\hline$p$ value & 0.971 & 0.226 & 0.297 & 0.578 & 0.089 & 0.062 & 0.077 & 0.051 \\
\hline
\end{tabular}

$p$ value calculated by ANOVA test.

1: I-gel group, 2: LMA group, 3: Tracheal tube group.

${ }^{*}$ Heart Rate, ${ }^{\dagger}$ SBP: systolic blood pressure, ${ }^{\dagger}$ DBP: diastolic blood pressure, ${ }^{\S} \mathrm{MAP}$ : mean arterial pressure. 
Table 3: Ventilatory parameters (inhaled and exhaled tidal volume, leak volume and minute volume in the study groups.

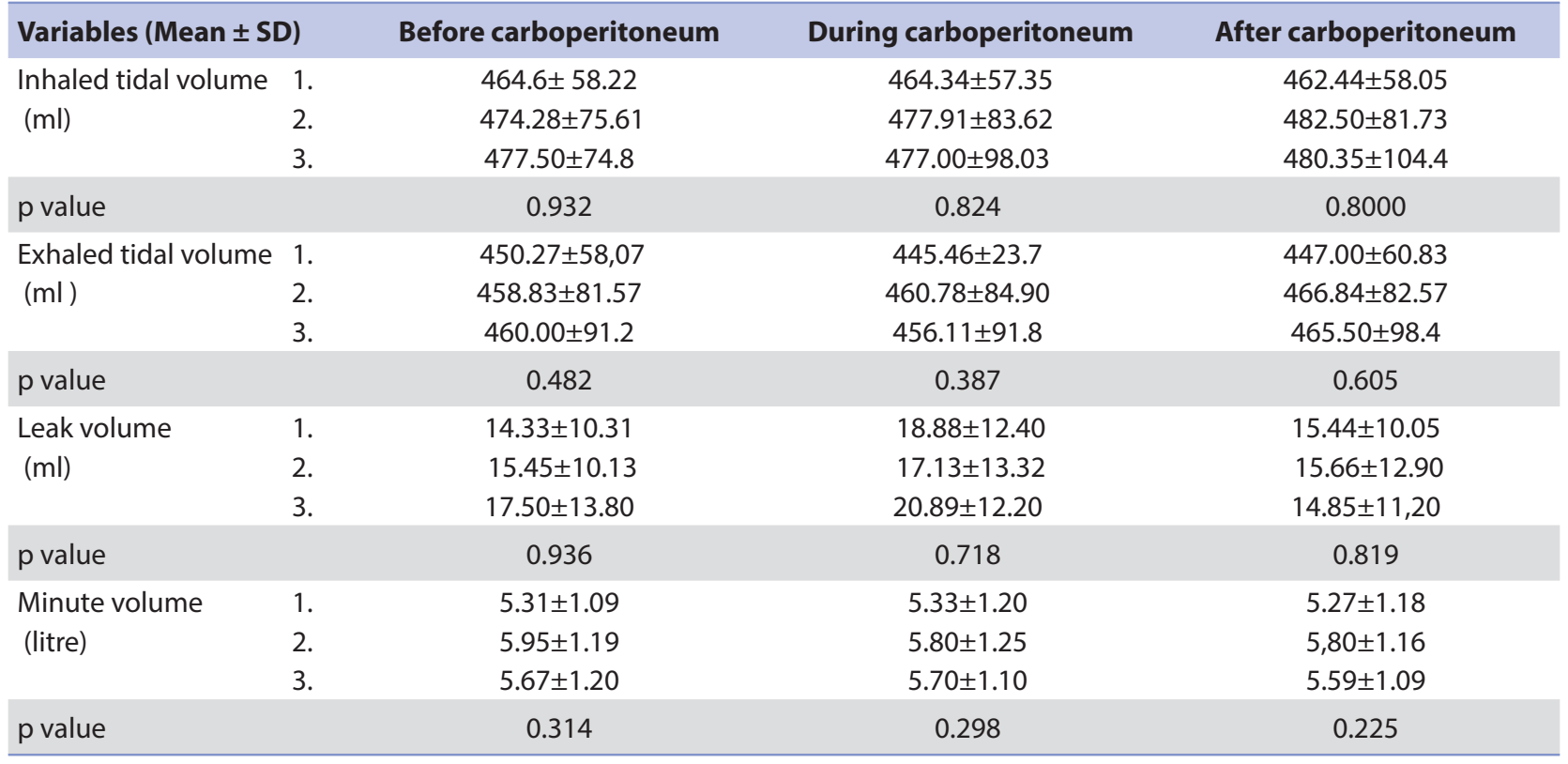

p value calculated by ANOVA test.

1: I-gel group, 2: LMA group, 3: Tracheal tube group

Table 4: Airway pressure, oxygen saturation and end tidal $\mathrm{CO}_{2}$ in the three groups.

\begin{tabular}{|c|c|c|c|c|}
\hline \multicolumn{2}{|c|}{ Variables (Mean \pm SD) } & \multirow{2}{*}{$\begin{array}{l}\text { Before carboperitoneum } \\
\qquad 13.96 \pm 2.73\end{array}$} & \multirow{2}{*}{$\begin{array}{l}\text { During carboperitoneum } \\
\qquad 20.11 \pm 3.46\end{array}$} & \multirow{2}{*}{$\begin{array}{c}\text { After carboperitoneum } \\
\qquad 14.46 \pm 2.88\end{array}$} \\
\hline Airway pressure & 1. & & & \\
\hline$\left(\mathrm{cmH}_{2} \mathrm{O}\right)$ & 2. & $13.29 \pm 2.35$ & $20.24 \pm 4.42$ & $15.37 \pm 3.36$ \\
\hline & 3. & $12.80 \pm 2.76$ & $19.05 \pm 4.82$ & $14.85 \pm 3.23$ \\
\hline \multicolumn{2}{|l|}{$p$ value } & 0.923 & 0.609 & 0.569 \\
\hline \multicolumn{2}{|c|}{ Oxygen saturation 1.} & $99.46 \pm 0.62$ & $99.82 \pm 0.38$ & $99.96 \pm 0.17$ \\
\hline \multirow[t]{2}{*}{$(\%)$} & 2. & $99.40 \pm 0.79$ & $99.93 \pm 0.25$ & $99.88 \pm 0.58$ \\
\hline & 3. & $99.9 \pm 0.23$ & $99.85 \pm 0.37$ & $100.00 \pm 0.00$ \\
\hline$p$ value & & 0.711 & 0.701 & 0.524 \\
\hline \multirow{3}{*}{$\begin{array}{l}\text { End tidal } \mathrm{CO}_{2} \\
(\mathrm{mmHg})\end{array}$} & 1. & $30.47 \pm 3.91$ & $34.44 \pm 3.75$ & $34.55 \pm 3.10$ \\
\hline & 2. & $31.81 \pm 3.87$ & $34.33 \pm 2.82$ & $34.07 \pm 2.77$ \\
\hline & 3. & $31.90 \pm 1.55$ & $34.78 \pm 2.63$ & $34.94 \pm 2.06$ \\
\hline \multicolumn{2}{|l|}{$p$ value } & 0.894 & 0.886 & 0.564 \\
\hline
\end{tabular}

$p$ value calculated by ANOVA test.

1: I-gel group, 2: LMA group, 3: Tracheal tube group.

\section{DISCUSSION}

This is a prospective interventional comparative study among the three airway devices (I-gel, classic LMA or tracheal tube) used during general anaesthesia with positive pressure ventilation for laparoscopic cholecystectomy. This study revealed that I-gel produces the least haemodynamic perturbations among the three devices with acceptable oxygenation and ventilation and all three devices can be used for patients with moderate airway pressure during general anaesthesia.
During supraglottic airway device use; especially with l-gel use, there was no clinically significant increment in heart rate and blood pressure after airway placement and removal but there was significant increment in these parameters after carboperitoneum. During LMA and tracheal tube use, there is perilaryngeal ant tracheal mucosa irritation which causes increase in heart rate and blood pressure and has additive effect on stress response and its consequences. 
The haemodynamic and ventilatory responses with I-gel, laryngeal mask airway and tracheal intubation during ....

Anotherimportantaspectis thatprobablythereisminimal stress response and haemodynamic perturbation with I-gel use which, being minimal, is completely blunted by use of anaesthetics during general anaesthesia but not in two other mentioned airway device use. This beneficial effect can be utilized during general anaesthesia with mechanical ventilation in patients with possibility of haemodynamic perturbations but with normal lung compliance. One must maintain adequate anaesthetic depth and do necessary monitoring during use of airway device to minimize complications and failure of the device.

Tracheal intubation is the gold standard for maintaining airway and delivering inhalation anaesthetic during general anaesthesia. But it induces haemodynamic stress response due to manipulation of pharynx, larynx and trachea during laryngoscopy and intubation because of the surge of catecholamine and cortisol despite different minimizing measures ${ }^{5,15}$. As laryngoscopy and tracheal stimulation is avoided during supraglottic airway device use, these devices can be used in selected elective cases during general anaesthesia to decrease these unwanted effects $^{8,10,13}$

Maltby et al assessed if classic LMA and Proseal LMA were good alternatives to tracheal intubation in laparoscopic gynaecological procedures with respect to pulmonary ventilation. There was no significant difference noted in airway pressure, oxygen saturation and end tidal carbon dioxide pressure before and during peritoneal insufflations. They recommended that tracheal tube could safely be substituted with correctly placed LMA and Proseal LMA during gynaecological laparoscopy ${ }^{3}$. In present study as well, classic LMA or I-gel were used as effectively as tracheal intubation in laparoscopic surgery.

Agrawal $\mathrm{G}$ et al reported that there was significantly high rise in heart rate and mean arterial pressure from base line in paediatric patients with use of LMA and tracheal tube$^{2}$. There was significant increase in heart rate and blood pressure in tracheal tube group compared to LMA group of patients. Jindal P et al compared haemodynamic effects of three supraglottic airway devices I-gel, LMA and streamlined pharyngeal airway (SLIPA) during general anaesthesia with muscle relaxation and I-gel revealed least hemodynamic changes during the device use ${ }^{4}$. Present study also shows similar findings of more stress response with tracheal tube compared to LMA and I-gel.

Won Jung Shin et al made a comparative study among I-gel, proseal LMA and classic LMA during general anaesthesia and didn't find any significant differences in haemodynamics and ventilatory responses ${ }^{8}$. Handan $G$ et al made a comparative study between proseal LMA and tracheal tube during laparoscopic cholecystectomy. Haemodynamic parameters and blood catecholamine and cortisol levels were compared between the two groups in intraoperative period. They reported that haemodynamic parameters were similar but cortisol level was higher after intubation and carboperitoneum in tracheal tube group and concluded that LMA can be safely used during laparoscopic surgery ${ }^{5}$.

Montazari $\mathrm{K}$ et al compared haemodynamic changes after use of facemask, LMA and tracheal tube and demonstrated that in healthy normotensive patients, the use of LMA for the airway management during general anaesthesia results in smaller cardiovascular changes than tracheal intubation ${ }^{6}$. Present study also revealed less haemodynamic response with LMA and l-gel compared to tracheal intubation.

Sing DK et al studied the haemodynamic parameters during insertion and removal of LMA and intubating LMA during airway management. They advised that LMA may offer some advantage over intubating LMA in patients where minimal changes in hemodynamics are desirable like coronary artery disease and cerebrovascular diseases ${ }^{9}$. Oczenski et al reported that the cardiovascular responses induced by laryngoscopy and intubation were more than twice as high as those produced by the insertion of an $\mathrm{LMA}^{15}$.

Ismail SA et al compared intraocular pressure, heart rate, systolic and diastolic blood pressure before and after use of I-gel, LMA and tracheal tube. Use of LMA and tracheal tube resulted in significant increase in intraocular pressure and hemodynamics compared to I-gel ${ }^{7}$. Present study also revealed that there is increase in heart rate and blood pressure compared to baseline and intergroup comparison also resulted in maximum response with tracheal tube and least with I-gel use.

Uppal V et al compared I-gel with cuffed tracheal tube during pressure controlled ventilation and reported that there was no significant difference between the leak volume and leak fraction of I-gel and the tracheal tube at moderate airway pressure mechanical ventilation and with hemodynamic stability'. We did volume control ventilation as there was no provision of pressure control mode in the anaesthesia machine and all three devices adopted moderate pressure during carboperitoneum.

Maltby JR, Watson NC et al compared classic LMA with tracheal tube during laparoscopic cholecystectomy 
regarding gastric distension and ventilation parameters. They reported that the incidence of gastric distension was similar in both groups, ventilation and oxygenation were well maintained and airway pressure similarly maintained $^{13}$. Our study also revealed adequate oxygenation, ventilation and acceptable leak volume in all three airway devices.

Sharma B et al have done comparative evaluation of I-gel with proseal LMA on respiratory mechanics during laparoscopic cholecystectomy and concluded that Proseal LMA formed a better seal while the dynamic compliance was higher with I-gel. Both devices provided optimal ventilation and oxygenation with least hemodynamic perturbations with $\mathrm{I}_{\text {-gel }}{ }^{16}$. Present study also reveals stable haemodynamics, oxygenation and ventilation with tracheal tube, classic LMA and I-gel during laparoscopic surgery and any one can be safely used in elective cases.

\section{REFERENCES}

1. Uppal V, Fletcher G, Kinsella J. Comparison of the I-gel with the cuffed tracheal tube during pressurecontrolled ventilation. BJA. 2009;102:264-8.

2. Agrawal G, Agrawal M, Taneja S. A randomized comparative study of intraocular pressure and hemodynamic changes on insertion of proseal LMA and conventional tracheal intubation in pediatric patients. J Anesthesiol Clin Pharmacol. 2012;28:326-9.

3. Maltby JR, Berault MT, Watson NC. LMA classic and LMA proseal are effective alternatives to endotracheal intubation for gynaecological laparoscopy. Can J Anesth. 2003;50(1)71-7.

4. Jindal $P$, Aslam $R$, Sharma JP. Is I gel a new revolution among supraglottic airway devices? A comparative evaluation. MEJ anaesthesia. 2009;20(1).

5. Handan G, Türkay Ç, Halil Y, Aytül SK, Hülya B. Comparison of hemodynamic and metabolic stress response caused by endotracheal tube and proseal LMA in laparoscopic cholecystectomy. J Res Med Sci. 2012;17(2)148-53.

6. Montazari K, Naghibi K, Hashemi SJ. Comparison of hemodynamic changes after insertion of LMA, face mask and endotracheal intubation. Acta Medica Iranica. 2004;42(6):432-40.

7. Ismail SA, Bisher NA, Kandil HW, Mowafi HA, Atawia HA. Intraocular pressure and hemodynamic response to I gel, LMA or endotracheal tube. Eur J Anesthesiol. 2011;28(6):443-8.

8. Shin WJ, Cheon YS. The supraglottic airway I gel in comparison with proseal LMA and classic LMA in anesthetized patients. Eur J anesthesiol. 2010;27:598-601.
Present study evaluated the haemodynamic and ventilatory parameters only and definitive stress response markers like catecholamines and cortisol assays were not done and large scale study is needed to draw definitive conclusion. This study also does not take into consideration leak pressure of individual airway devices and other unwanted effects like sore throat, trauma, gastric distension and aspiration.

\section{CONCLUSION}

As LMA produces minimal and I-gel the least haemodynamic response compared to tracheal intubation and other advantages as well, these supraglottic devices can be used in selected elective surgical cases where these stress responses may be undesirable and better avoided. Ventilatory parameters during carboperitoneum are comparable in all three devices and LMA or I-gel can be used safely with proper placement and monitoring.

9. Singh DK, Jindal P. Comparative evaluation of hemodynamic changes during insertion and removal of LMA and ILMA. Internet J Anesthesiol. 2006;11(1).

10. Uppal V, Gangaiah S, Fletcher G, Kinsella J. Randomized crossover comparison between the I-gel and the LMA-Unique in anaesthetized, paralyzed adults. BJA. 2009;103:882-5.

11. Singh I, Gupta M. Comparison of clinical performances of I gel with LMA ProSeal in elective surgeries. Indian J Anesth. 2009;53(3):302-5.

12. Weber U, Oguz R, Potura Kimberger LO, Kober A, Tschernko E. Comparison of the I gel and the LMA Unique in patients with mild to moderate obesity during elective short term surgery. Anesthesia. 2011;66(6):481-7.

13. Maltby JR, Beriault MT, Watson NC, Liepert DJ, Fick GH. Gastric distension and ventilation during laparoscopic cholecystectomy: LMA classic vs. tracheal intubation. Can J Anesth. 2000 Jul;4(7):622-6.

14. Braude N, Clements EA, Hodges UM, Andrews $B P$. The pressure response and laryngeal mask insertion. A comparison with tracheal intubation. Anaesthesia. 1989 Jul;44(7):551-4.

15. Oczenski W, Krenn $H$, Dahaba $A A$, Binder $M$, Jellinek $H$, Schwarz $S$, et al. Hemodynamic and catecholamine stress responses to insertion of the Combitube, laryngeal mask airway or tracheal intubation. Anesth Analg. 1999 Jun;88(6):1389-94.

16. Sharma B, Sehgal R, Chand S, Jayashree S. PLMA vs. I-gel: A comparative evaluation of respiratory mechanics in laparoscopic cholecystectomy. J clin Anaesth. 2010;26(4):451-7. 